\title{
Cem anos da Entomologia Forense no Brasil (1908-2008)
}

\author{
José Roberto Pujol-Luz ${ }^{1}$, Luciano Chaves Arantes ${ }^{1,2} \&$ Reginaldo Constantino $^{1}$
} ${ }^{1}$ Núcleo de Entomologia Urbana e Forense, Departamento de Zoologia, Instituto de Ciências Biológicas, Universidade de Brasília. Brasília, DF.
70910-900. jrpujol@ @unb.br
${ }^{2}$ Seção de Perícias e Análises Laboratoriais, Instituto de Criminalística, Polícia Civil do Distrito Federal. SAISO, Complexo da Polícia Civil, bloco
E, Brasília-DF. 70610-200. lca1969@gmail.com

\begin{abstract}
One hundred years of forensic entomology in Brazil (1908-2008). The history of the development of forensic entomology in Brazil, its current status and perspectives are reviewed. Those studies in Brazil began in 1908 with the pioneer works conducted by Roquette-Pinto and Oscar Freire, who noted the high diversity of the native fauna of necrophagous insects and the impossibility of direct application of the methods developed in Europe. In the last two decades, forensic entomology advanced rapidly in Brazil, but there still are some important limitations, especially in relation to the taxonomy, biology and ecology of necrophagous insects and the lack of interaction between researchers and police investigators. Today there are in Brazil over 20 researchers involved with forensic entomology and tens of police investigators with some training in this field. Guidelines for the development of this field of investigation in Brazil are also presented.
\end{abstract}

KEYWORDS. Forensic taphonomy; history; necrophagous insects.

\begin{abstract}
RESUMO. Cem anos da Entomologia Forense no Brasil (1908-2008). Apresentamos uma história do desenvolvimento da Entomologia Forense no Brasil e uma avaliação do estado da arte e perspectivas. Esses estudos no Brasil iniciaram-se em 1908 com os trabalhos pioneiros de Roquette-Pinto e Oscar Freire, que notaram a grande diversidade da fauna de insetos necrófagos e a impossibilidade de aplicação direta de métodos desenvolvidos na Europa. Nas últimas duas décadas a Entomologia Forense tem avançado rapidamente no Brasil, mas ainda existem lacunas importantes no conhecimento, especialmente com relação à taxonomia, biologia e ecologia dos principais grupos de moscas e besouros necrófagos e também falta de integração entre os entomologistas e a polícia judiciária. Atualmente existem no Brasil mais de 20 pesquisadores desenvolvendo pesquisas relacionadas com Entomologia Forense e algumas dezenas de peritos criminais com treinamento nessa área, em quase todos os estados brasileiros. Neste trabalho são também apresentadas algumas diretrizes para políticas de desenvolvimento deste campo de pesquisas no Brasil.
\end{abstract}

PALAVRAS-CHAVE. História; insetos necrófagos; tafonomia forense.

A Entomologia Forense - o estudo de insetos e outros artrópodes associados a diversas questões criminais - serve como uma ferramenta auxiliar, por exemplo, na investigação de crimes contra pessoas vítimas de morte violenta.

Esses estudos iniciaram-se no Brasil em 1908, com os trabalhos pioneiros de Edgard Roquette Pinto e Oscar Freire, respectivamente nos Estados do Rio de Janeiro e da Bahia. Com base em estudos de casos em humanos e animais realizados na primeira década do Século XX, esses autores registraram a diversidade da fauna de insetos necrófagos em regiões de Mata Atlântica, então ainda bastante preservadas. Tais trabalhos foram realizados pouco tempo depois da publicação do livro de Mégnin (1894), o primeiro a tratar do tema de forma sistemática, e chamaram a atenção por postura crítica e seu esforço em desenvolver métodos adequados às condições locais do Brasil.

O desenvolvimento da Entomologia Forense no Brasil tem sido facilitado pela sólida tradição brasileira no estudo de insetos das Ordens Diptera e Coleoptera. Outros grupos de insetos também são relevantes para a Entomologia Forense, mas as moscas e os besouros são os mais importantes, especialmente em casos envolvendo morte. Devido à sua importância na medicina, na saúde pública, na veterinária e na agricultura, moscas e besouros foram extensamente estudados por profissionais de diversas áreas do conhecimento. Além disso, as coleções taxonômicas brasileiras contêm uma razoável representação da diversidade de insetos de nossa região. Existem obviamente ainda lacunas importantes, principalmente a escassez de informações sobre a biologia, ecologia e distribuição geográfica das espécies necrófagas.

O entomologista forense deve possuir um bom conhecimento de taxonomia, biologia e ecologia de insetos. Esse perfil é relativamente raro, mas felizmente o Brasil possui um bom número de especialistas aptos para conduzir pesquisas e treinar profissionais nessas áreas do conhecimento, não só no estudo das moscas e besouros, mas também em outros grupos de animais necrófagos ou associados ao processo de decomposição cadavérica.

Estudos sobre a biologia, ciclo de vida e ecologia de insetos necrófagos são ainda incipientes, devido à sua complexidade, alto custo e demora na obtenção de resultados. O conhecimento taxonômico dos dípteros e coleópteros necrófagos é essencial para a Entomologia Forense, mas não suficiente. A estimativa do intervalo de morte, por exemplo, depende também de informações ecológicas e biológicas, especialmente sobre o desenvolvimento pós-embrionário de 
espécies das famílias Calliphoridae, Muscidae, Sarcophagidae e Stratiomyidae entre as moscas e Dermestidae, Cleridae, Histeridae e Scarabaeidae, entre os besouros.

Neste trabalho apresentamos uma revisão sobre a história do desenvolvimento da Entomologia Forense no Brasil desde o início do século XX e uma avaliação do estado da arte e perspectivas desta ciência em nosso país.

Um breve histórico sobre a Entomologia Forense. Nas civilizações antigas da Babilônia e do Egito, as moscas aparecem como amuletos, como deuses (Baal Zebub, O Senhor das Moscas) e como uma das pragas na história bíblica do Êxodo (Thompson \& Pont 1993).

A metamorfose das moscas já era conhecida no antigo Egito. Um papiro encontrado no interior da boca de uma múmia continha a seguinte inscrição: "As larvas não se transformarão em moscas dentro de ti" [Papiro de Gizé no. 18026:4:14]. A maioria dos insetos encontrados em corpos embalsamados são os mesmos que agora auxiliam na solução de casos de morte (Greenberg 1991).

O primeiro caso documentado de Entomologia Forense está relatado em um manual de Medicina Legal Chinês do Século XIII. Foi um caso de homicídio em que um lavrador apareceu degolado por uma foice. Para resolver o caso, todos os lavradores da região foram obrigados a depositar suas foices no solo, ao ar livre. As moscas pousaram em apenas uma delas, atraídas pelos restos de sangue que ainda estavam aderidos à lâmina. A conclusão foi de que aquela era a foice do assassino (Benecke 2001).

A primeira estimativa de intervalo post-mortem baseada em insetos foi feita pelo médico francês Bergeret (1855), mas o primeiro livro sobre o tema foi "La faune de cadavres" de Mégnin (1894), no qual o autor inclui fundamentação teórica, descrições dos insetos e relatos de casos reais estudados por ele e colaboradores.

Apesar dos estudos de Mégnin (1894), a Entomologia Forense foi negligenciada por muito tempo, pela falta de entomologistas especializados no estudo da fauna cadavérica em todo o mundo e principalmente por causa do distanciamento entre entomologistas e profissionais da criminalística (médicos-legistas e peritos criminais). O interesse só foi retomado na segunda metade do Século XX. Leclercq (1969) publicou "Entomology and Legal Medicine" e posteriormente Smith (1986) publicou o livro "A Manual of Forensic Entomology". No final do século XX sua aplicação tornou-se rotina, especialmente na América do Norte e na Europa, onde muitos grupos de pesquisa têm se dedicado ao estudo desse tema.

A Entomologia Forense no Brasil. No Brasil, o marco inicial da entomologia forense está associado ao trabalho de Oscar Freire (Fig. 1); em 1908, apenas quatorze anos após a publicação do trabalho de Mégnin (1894), apresentou à Sociedade Médica da Bahia a primeira coleção de insetos necrófagos e os resultados de suas investigações, em grande parte obtidas em estudos com cadáveres humanos e de pequenos animais.

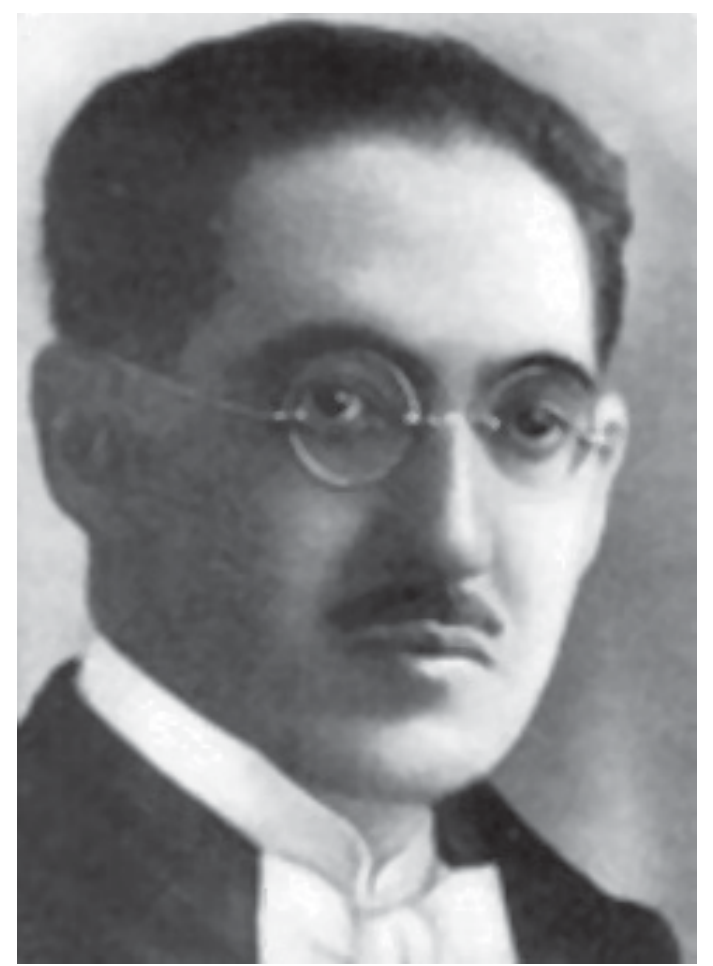

Fig. 1. Oscar Freire.

(Fonte:http://www.hcnet.usp.br/historiahc/imagens/pessoas/ dr_oscar_freire.jpg)

Antes dele, Domingos Freire (Factos da vida dos insetos. II. Fauna dos cadáveres, 1908) publicara um artigo sobre o tema, criticado por Freire como um reflexo impessoal do trabalho de Mégnin. No mesmo ano, Roquette-Pinto (Fig. 2) publicou um estudo de caso "Nota sobre a fauna cadavérica no Rio de Janeiro" também com base em um cadáver humano (RoquetePinto 1908). Esses dois pesquisadores também lançaram as bases da Entomologia Forense nos trópicos. Seguiram-se a eles, entre 1911 e 1941, os trabalhos de Herman Lüderwaldt (Fig. 3), Samuel Pessôa (Fig. 4) e Frederico Lane (Fig. 5), descrevendo especialmente a fauna de besouros escarabeídeos necrófagos do Estado de São Paulo.

Lüderwaldt (1911) estudou os besouros da coleção do então Museu Paulista, atual Museu de Zoologia da Universidade de São Paulo, e publicou uma lista de insetos necrófagos. Segundo Oscar Freire, esse trabalho foi deficiente em relação aos dípteros, mas "era uma valiosa contribuição sobre os coleópteros da fauna cadavérica, embora não cuidasse com a devida atenção do ponto de vista médico-legal" (Pessôa \& Lane 1941). Uma característica notável do trabalho de Lüderwaldt foi o cuidado em comparar a fauna de besouros do Brasil com a da Alemanha. Seus resultados apontaram para a mesma direção dos de Oscar Freire no referente à diversidade dos insetos necrófagos nos trópicos. Suas observações minuciosas, baseadas em experimentos sobre a biologia e o comportamento de besouros necrófagos em atividade nas carcaças, realizados no Horto do Museu Paulista, são ainda hoje uma referência importante sobre o tema.

Freire (1914a, 1914b) publicou ainda "Algumas notas para 
o estudo da fauna cadavérica da Bahia", estudando um grande número de dípteros, com boa contribuição ao estudo médicolegal e fez importantes críticas ao método proposto por Mégnin.

Em 1919, Belfort de Mattos, sob a orientação de Oscar Freire, escreveu um trabalho sobre os sarcofagídeos de São Paulo (Mattos 1919; Pessôa \& Lane 1941).

O conjunto da obra de Oscar Freire sobre a "Fauna cadavérica brasileira" foi publicado postumamente em 1923 na Revista de Medicina (Freire 1923; Pessôa \& Lane 1941). O conhecimento acumulado por Oscar Freire e sua experiência ao longo dos anos serviram de modelo para os estudos atuais sobre a Entomologia Forense no Brasil, principalmente a preocupação em trazer soluções às questões médico-legais, especialmente ao problema da cronotanatognose, a estimativa do tempo de morte; criticou o trabalho de Mégnin (1894), que considerou "excessivamente teórico e esquemático"; concordou que existe certo padrão de sucessão ou seriação (ondas ou legiões de trabalhadores da morte) no modo pelo qual os insetos visitam o cadáver, mas chamou atenção para o fato de que essa "ordem" é apenas freqüente e "não constante, nem imutável"; também chamou atenção para as muitas exceções e acrescentou que:

(1) Não há exclusivismo de espécies de insetos para cada fase da putrefação;

(2) É fator de importância a concorrência vital entre os necrófagos;

(3) Influi na sua presença ou na sua ausência a riqueza em espécies e gêneros da região, a distribuição "topográfica" (geográfica);

(4) Não há isocronismo dos períodos da decomposição cadavérica, e

(5) Uma cronologia precisa é impossível (Freire 1914b, 1923; Pessôa \& Lane 1941).

O rigor científico das observações e críticas de Oscar Freire vem ao encontro da visão atual de que as técnicas desenvolvidas em outros países não podem ser diretamente aplicadas no Brasil, pelas diferenças na entomofauna e no clima. O Brasil é o país com a maior biodiversidade do mundo e isso se reflete também na fauna associada a cadáveres. Além disso, cada bioma tem sua fauna e condições locais próprias, o que exige o estudo das entomofaunas regionais, principalmente dípteros e besouros e seus padrões de sucessão em cadáveres, antes da aplicação das técnicas de entomologia forense. As estimativas devem ser aferidas de acordo com os padrões regionais e locais. As pesquisas nesse sentido ainda são incipientes, limitando a aplicação da Entomologia Forense no Brasil.

Esse primeiro período se encerra com a magnífica obra de Pessôa \& Lane (1941) sobre os coleópteros de interesse médico-legal do Estado de São Paulo. Os autores trataram especialmente da família Scarabaeidae, mas também apresentaram nesse artigo um histórico detalhado das pesquisas sobre o tema, no Brasil e no mundo, até aquela data. Importantes dados sobre a taxonomia e biologia dos besouros necrófagos foram fornecidos.

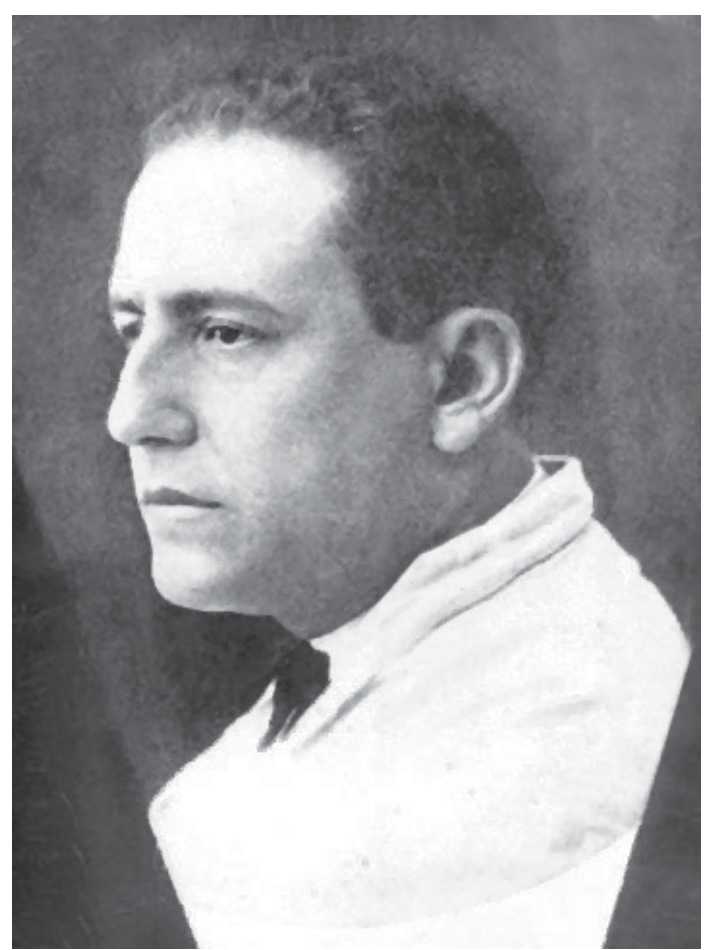

Fig. 2. Edgard Roquette-Pinto.

(Fonte: http://www.museunacional.ufrj.brsae.html)

Foram bastante raros os trabalhos científicos sobre entomologia forense ou a ela relacionados, entre as décadas de 1940 a 1980. O período imediatamente posterior também se caracterizou pelo desenvolvimento e ações dos pesquisadores brasileiros nos estudos sobre taxonomia, biologia, desenvolvimento pós-embrionário e ecologia, especialmente de dípteros necrobiontófagos (e.g. Guimarães et al. 1983; Monteiro-Filho \& Penereiro 1987; Salviano et al. 1996). O trabalho exploratório de Monteiro-Filho \& Penereiro (1987) sobre a sucessão de insetos sobre uma carcaça de roedor no Estado de São Paulo caracteriza o retorno ao estudo do tema.

Os estudos sobre taxonomia, biologia e ecologia de dípteros necrófagos e biontófagos no Brasil, estudos básicos para a Entomologia Forense, têm sido realizados por vários pesquisadores brasileiros, sendo notáveis os trabalhos de Hugo de Souza Lopes (Sarcophagidae), Rubens Pinto de Mello (Calliphoridae), José Henrique Guimarães e Nelson Papavero (Calliphoridae).

Os primeiros trabalhos sistemáticos sobre entomologia forense abordando estudos sobre a diversidade, ecologia, taxonomia e sucessão da fauna cadavérica foram desenvolvidos por Arício Xavier Linhares, Claudio José Barros de Carvalho e respectivas equipes, como por exemplo: Souza \& Linhares (1997); Moura et al. (1997); Carvalho et al. (2000); Carvalho \& Mello-Patiu (2008), sendo estes dois pesquisadores os responsáveis pelo esforço de desenvolver uma Entomologia Forense genuinamente brasileira.

No entanto, a consolidação da Entomologia Forense no 
Brasil ainda depende de um ponto importante: a interação dos trabalhos acadêmicos com a realidade da demanda da polícia judiciária. O livro de Janyra Oliveira-Costa (2003) foi o primeiro no Brasil a compilar a informação disponível na literatura nacional e internacional, contribuindo para divulgar a fundamentação teórica e para uniformizar a linguagem entre o pesquisador e o perito criminal. Seguindo a mesma linha, Miranda et al. (2006) publicaram um manual de coleta de amostras de insetos em locais de crimes. Ainda assim, foram publicados no Brasil poucos estudos de casos reais, caracterizando essa interação entre a academia e a polícia (e.g . Oliveira-Costa \& Lopes 1999; Oliveira-Costa \& Mello-Patiu 2004; Pujol-Luz et al. 2006, 2008).

Tarefas da Entomologia Forense. A Entomologia Forense dedica-se à aplicação do estudo dos insetos na solução de casos criminais e disputas judiciais. Insetos podem ser usados como evidência na solução de crimes e, em alguns casos, podem estar no centro de disputas judiciais ao causar danos a produtos armazenados ou estruturas (Keh 1985).

A Entomologia Forense foi classificada em três subáreas, descritas abaixo (modificado de Lord \& Stevesson 1986).

1) Urbana: relativa às ações cíveis envolvendo a presença de insetos em bens culturais, imóveis ou estruturas. Um caso típico seria o do comprador de um imóvel que, pouco tempo depois da compra, descobre que ele se encontra infestado por cupins e responsabiliza o vendedor pelo seu prejuízo. A questão a ser respondida pela Entomologia Forense é o tempo de infestação e se ocorreu antes ou depois da compra.

2) Produtos armazenados: diz respeito à contaminação, em pequena ou grande extensão, de produtos comerciais estocados. O comprador do lote de alimento infestado por insetos pragas pode exigir do vendedor uma compensação pelo prejuízo. O desafio para a Entomologia Forense seria determinar quando ocorreu a infestação.

3) Médico-legal: refere-se a casos de morte violenta (crime contra a pessoa, acidentes de massa, genocídios, etc.). A principal contribuição da Entomologia Forense, nesses casos, é a estimativa do intervalo post-mortem.

O conhecimento acerca da ecologia, biologia e distribuição dos insetos já contribuiu para a solução de crimes informando quando, onde, por quem e como o crime foi cometido. Além da contribuição mais importante e recorrente - a estimativa do intervalo post-mortem - insetos já foram utilizados como indícios de casos de movimentação de cadáveres, na reconstituição da movimentação de veículos, na localização de região produtora de drogas com conseqüente identificação da rede de distribuição (Crosby et al. 1986), na confirmação de hipótese de abandono de menores e maus tratos a idosos (Benecke et al. 2004; Benecke \& Lessig 2001), na identificação de autoria do crime por meio do DNA obtido do sangue ingerido por insetos hematófagos ou de suas fezes (Repogle et al. 1994; Mumcuoglu et al. 2004) e na detecção de drogas, venenos, medicamentos e metais pesados em imaturos, adultos, pupários, exúvias e fezes de insetos (Amendt et al. 2004).

Além da estimativa do intervalo de morte, outra importante

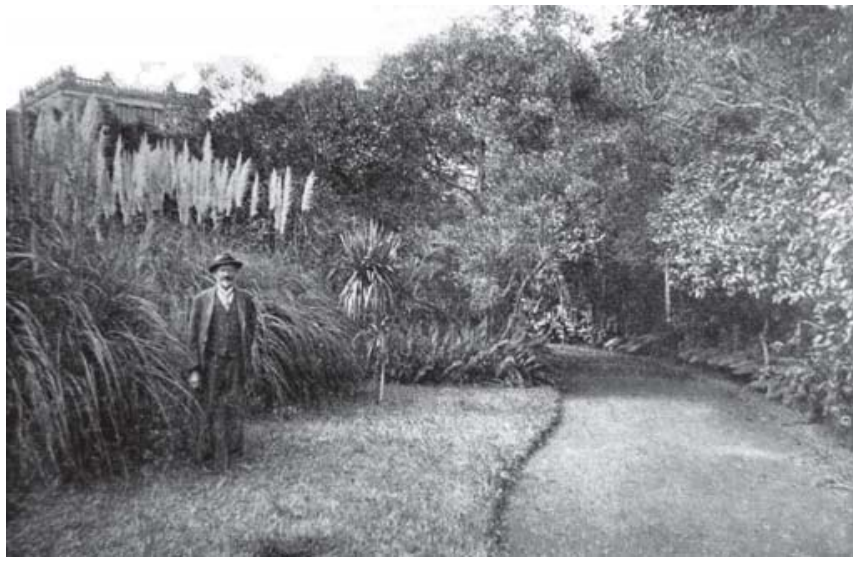

Fig. 3. Hermann Luederwaldt no Horto do Museu Paulista, onde realizou a maior parte das suas observações sobre besouros necrófagos.

contribuição da entomologia forense na investigação de cenas de crimes com mortes violentas é a avaliação e interpretação das evidências deixadas ou causadas por insetos. A ação de insetos sobre os restos humanos pode causar artefatos como a destruição de tecidos moles e traumas nos órgãos internos e externos e nas partes esqueletizadas do cadáver (Haskell et al. 1997).

O comportamento dos insetos sobre o cadáver (fonte primária de recursos para os animais necrófagos) pode causar o desmembramento dos restos mortais, ou o enterramento (ocultação) ou a exposição de partes do corpo. Estas atividades são importantes fatores a serem considerados na observação inicial da cena do crime. Morton \& Lord (2006) afirmaram que mesmo os vertebrados que normalmente podem ser responsáveis por eventual destruição de tecidos moles, desmembramentos e dispersão dos ossos, aguardam o término da atividade de insetos, especialmente a ação das larvas de moscas, para se alimentarem da carcaça.

$\mathrm{O}$ estudo da ecologia da decomposição, incluindo o entendimento dos processos de decomposição, dispersão, erosão, soterramento, exposição e preservação de um organismo morto é denominado Tafonomia (Efremov 1940). A Tafonomia Forense examina como as variáveis ambientais, bióticas e abióticas alteram as evidências que são objeto de uma investigação criminal (Haglund \& Sorg 1997; Sorg \& Haglund 2002; Morton \& Lord 2006; Carter et al. 2007). Os dípteros se destacam como agentes biotafonômicos nas fases iniciais do processo de decomposição, sendo os principais responsáveis pelo consumo das partes moles.

A reconstrução dos processos sofridos pelo cadáver nos períodos peri-mortem e post-mortem são fatores importantes para diferenciar as alterações produzidas por fenômenos naturais daquelas provocadas pelo evento que causou a morte (Ubelaker 1997). Mesmo pequenas alterações post-mortem, se não forem identificadas como causadas por algum tipo de inseto, podem ser confundidas com lesões ou artefatos produzidos pelo evento que causou a morte, podendo alterar as conclusões da perícia criminal (Ururahy-Rodrigues et al., 2008). 
O Estado da Arte da Entomologia Forense no Brasil. Nos últimos 10 anos, vários eventos foram importantes para congregar pesquisadores e peritos criminais interessados em Entomologia Forense.

Em 2003, o Simpósio "O Estado da Arte da Entomologia Forense no Brasil”, patrocinado pelo Instituto de Criminalística da Polícia Civil do Distrito Federal com a colaboração da Universidade de Brasília, reuniu cerca de 30 participantes, entre estudantes de graduação e pós-graduação, peritos criminais, agentes e delegados de polícia. Participaram como palestrantes peritos dos Institutos de Criminalística do Distrito Federal, do Rio de Janeiro e pesquisadores da Universidade de Brasília e da Universidade Estadual de Campinas.

Em 2004, durante o XXV Congresso Brasileiro de Zoologia, realizado em Brasília, ocorreu o primeiro Simpósio de Entomologia Forense no Brasil, com apoio do Ministério da Justiça, do Conselho Britânico e da Embaixada Britânica no Brasil. Aberto ao público em geral, aos profissionais da esfera da justiça e aos congressistas, esse evento contou com a participação de dois especialistas britânicos vinculados ao King's College de Londres e à Scotland Yard, compartilhando suas experiências e pesquisas.

Em 2007 foi criada a Associação Brasileira de Entomologia Forense (ABEF), sediada em Campinas, SP, simultaneamente com o I Simpósio Brasileiro sobre essa especialidade. O II Simpósio Brasileiro de Entomologia Forense ocorreu em 2008, durante o XXVII Congresso Brasileiro de Zoologia, em Curitiba, Paraná.

Tais eventos ajudaram a definir as divisões gerais da pesquisa em Entomologia Forense no Brasil. Diferentes grupos de pesquisadores, alguns consolidados e outros emergentes, concentram seus estudos em três grandes áreas: (1) Entomologia Forense sensu stricto (Urbana, Produtos Estocados e Médico-Legal); exemplos: estudos de simulações de cenas de crimes utilizando modelos de carcaças animais e da aplicação do conhecimento obtido em casos judiciais); (2) Ecologia da Decomposição e Tafonomia Forense; exemplos: estudos teóricos ou práticos sobre a sucessão da fauna cadavérica, estudos exploratórios sobre a diversidade de insetos necrófagos e sua participação nas ações de espalhar/ enterrar/soerguer o cadáver ou suas partes; (3) Biologia do Organismo'; exemplos: estudos sobre desenvolvimento pósembrionário e diferentes modelos e variações do comportamento dos insetos.

Mais de 20 pesquisadores em entomologia forense ligados a Instituições de Ensino Superior atuam hoje em dia em todas as regiões brasileiras (Fig. 6). Destacam-se ainda a formação e qualificação de dezenas de peritos e médicos-legistas de todas as regiões brasileiras em três Cursos Especiais de Entomologia Forense, promovidos pela ação conjunta do Ministério da Justiça, do Ministério da Ciência e Tecnologia e da Universidade de Brasília, onde foi desenvolvido o projeto do Centro Nacional de Entomologia Forense (2003-2005). Os Cursos foram ministrados por professores e especialistas da Universidade de Brasília, do Instituto de Criminalística da Polícia Civil do Distrito Federal, do Instituto Médico-Legal da

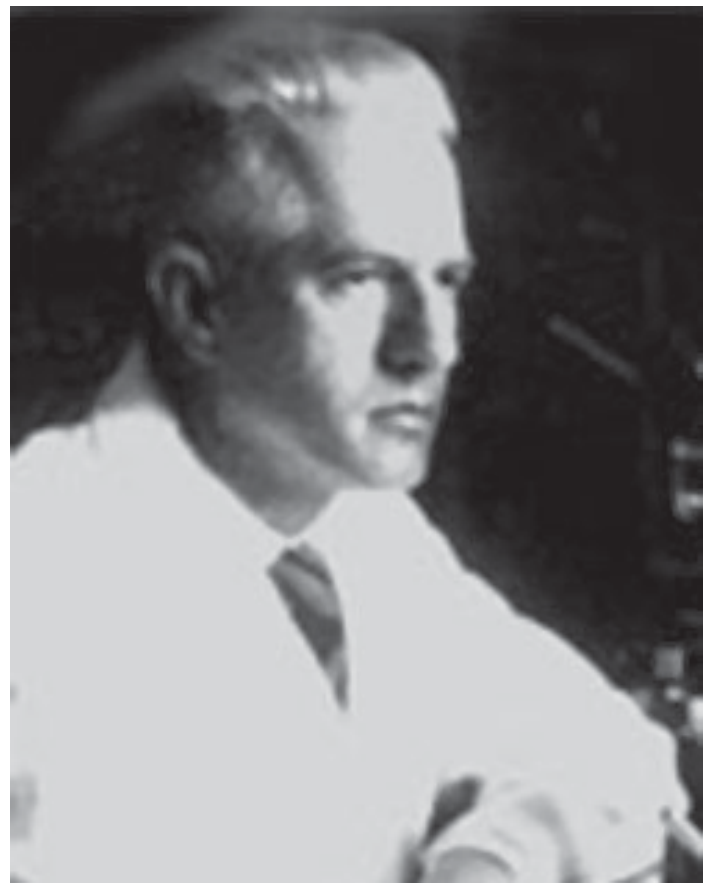

Fig. 4. Samuel Pessoa.

(Fonte: http://www.sbhm.org.br/index.asp?p=medicos_view\&codigo $=211$

Polícia Civil do Distrito Federal, da Universidade Federal Rural do Rio de Janeiro e do Instituto Carlos Éboli da Polícia Técnica do Estado do Rio de Janeiro.

Integração da Academia e Polícias Judiciárias. Um dos entraves para a expansão da utilização de evidências entomológicas como ferramenta auxiliar na solução de crimes é o distanciamento entre a academia e os profissionais das polícias judiciárias responsáveis pela produção de provas materiais a partir do exame de cenas de crime e de cadáveres os peritos criminais e os médicos legistas.

Conhecimentos produzidos por equipes compostas por entomologistas, peritos criminais e médicos legistas são escassos atualmente e certamente contribuirão enormemente para o desenvolvimento da entomologia forense no Brasil, pois irão associar o melhor de dois mundos: a experiência científica dos professores com a casuística dos peritos criminais e médico-legistas.

Com essa casuística, adquirida ao longo de anos em cenas de crime e/ou examinando cadáveres, perguntas relevantes são formuladas rotineiramente. Respostas a algumas delas, muitas com elevado peso para a solução de crimes, podem ser obtidas a partir de experimentos bem delineados em Entomologia Forense. Entretanto, o cenário atual, com excesso de trabalho e reduzido número de profissionais nas polícias técnico-científicas e nas universidades brasileiras, somado à falta de intimidade natural entre esses profissionais desestimula o desenvolvimento de pesquisas conjuntas.

Não obstante, as poucas oportunidades nas quais esses profissionais atuam conjuntamente resultaram na produção de provas materiais importantes. Como exemplo, citamos o 
Estado do Rio de Janeiro e o Distrito Federal, onde o vínculo entre a academia e a polícia judiciária tem contribuído para a incorporação da entomologia forense no rol das ciências utilizadas rotineiramente como ferramentas para produção de evidências.

Juntamente com ações de estímulo à pesquisa em entomologia forense e sua utilização rotineira pela polícia judiciária é necessário promover reuniões (ou a criação de um fórum temático) entre a comunidade acadêmica e a polícia técnico-científica, para estabelecer as questões prioritárias a serem abordadas. Um dos maiores desafios para o desenvolvimento futuro desta área do conhecimento é a associação entre os dados experimentais e a casuística forense (Amendt et al. 2004).

Como aconteceu com outras áreas das Ciências Forenses, a entomologia forense necessita estabelecer protocolos que garantam uma padronização mínima dos procedimentos adotados durante a coleta das evidências entomológicas e dos dados meteorológicos; a preservação, o transporte e a criação dos insetos imaturos; e a validação dos dados obtidos a partir de diferentes experimentos sobre a biologia dos insetos. Movimento nesse sentido já foi feito pela Associação Européia para a Entomologia Forense (EAFE, em inglês) ao publicar um artigo acerca das melhores práticas em entomologia forense com recomendações e condutas padrões a serem seguidas por policiais, patologistas e entomologistas (Amendt et al. 2007).

Em 2008 o governo brasileiro reconheceu a necessidade de investir neste setor do conhecimento e criou um Grupo de Trabalho vinculado à Secretaria Nacional de Segurança Pública do Ministério da Justiça. Esse grupo, sediado em Brasília, foi denominado "Rede Nacional de Entomologia Forense (ReNEF)" e é composto por 5 pesquisadores e 5 peritos criminais dos estados do Amapá, Amazonas, Bahia, São Paulo, Rio de Janeiro, Paraná e Distrito Federal.

A Rede Nacional de Entomologia Forense tem como metas primárias, (1) estimular e desenvolver as pesquisas sobre Entomologia Forense no Brasil; (2) fortalecer a cooperação/ integração entre as Universidades/Institutos de Pesquisa e a Polícia Judiciária e (3) fornecer as bases para a padronização de métodos e técnicas, criando instrumentos de jurisprudência quando os insetos forem objeto da investigação ou provas materiais.

Principais diretrizes e metas da Entomologia Forense no Brasil. Suas aplicações na solução de casos criminais depende de estudos básicos sobre taxonomia, biologia, ciclo de vida e ecologia dos insetos de potencial interesse forense. Isso inclui não apenas insetos necrófagos, mas também os insetos envolvidos em questões urbanas e ataques aos produtos armazenados. A seguir estão listadas as linhas prioritárias de pesquisa, desenvolvimento e ações nesse sentido:

(1) Identificação de insetos de interesse forense:

(a) Taxonomia dos grupos de interesse forense; sem estudos básicos, especialmente revisões e monografias, não é possível realizar identificações seguras;

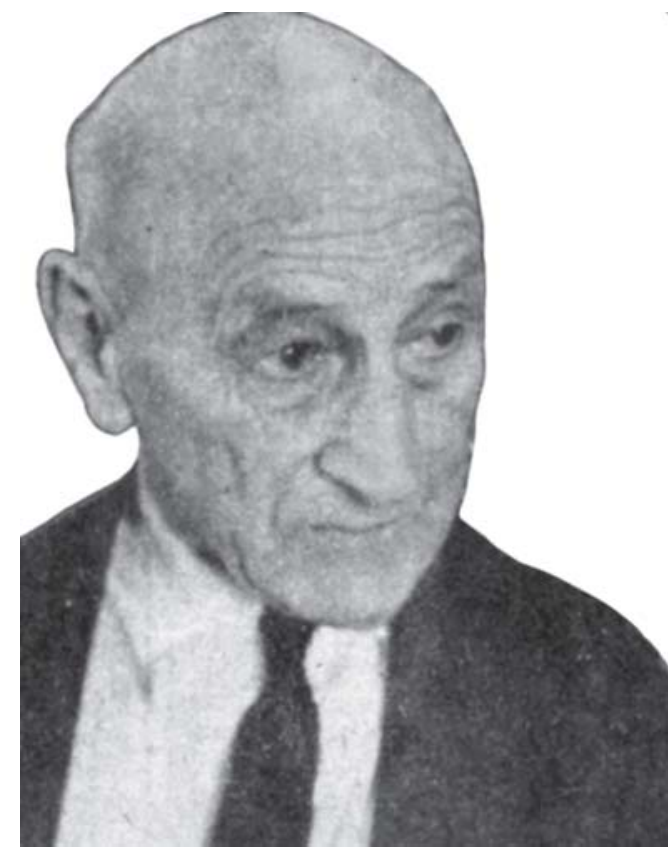

Fig. 5. Frederico Lane.

(b) Produção de guias e chaves de identificação dos insetos de interesse forense para uso rotineiro de peritos, produzidos por especialistas, tendo como público-alvo os peritos e testados para esse fim; deverão ser elaborados na forma de chaves eletrônicas interativas e bem ilustradas e sempre que possível publicados em periódicos especializados [Ver item (7) abaixo];

(c) Preparação de coleções de referência, com auxílio de taxonomistas, para uso em rotinas de identificação, disponíveis em todas as instituições; devem também conter representantes das espécies de interesse forense, identificados e organizados de modo a permitir identificação por comparação; o uso dessas coleções exige também treinamento prévio dos peritos.

(2) Estudos sobre biologia e ciclo de vida de insetos de interesse forense, principalmente dípteros e coleópteros das famílias Calliphoridae, Muscidae, Fanniidae, Sarcophagidae, Stratiomyidae, Dermestidae, Cleridae, Histeridae, Silphidae e Scarabaeidae:

(a) Ciclo de vida e tempo de desenvolvimento - estudos básicos sobre o tempo de desenvolvimento das espécies de interesse forense, da oviposição à emergência do adulto, são essenciais; é importante saber a duração média, máxima e mínima de cada etapa e cada ínstar para todas as espécies usadas, para estimativas do tempo de morte; é importante também a descrição morfológica de cada ínstar, para possibilitar sua identificação; na maioria dos casos, as informações disponíveis na literatura nessa área são precárias;

(b) Estudos sobre o efeito da temperatura sobre o desenvolvimento - havendo disponibilidade de dados de temperatura, é possível refinar a estimativa de tempo de desenvolvimento, levando em consideração esse fator, isto geralmente envolve o uso de graus-dia ou graus-hora 
acumulados; no entanto, é necessário estudar o efeito da temperatura sobre o ciclo de vida de todas as espécies e conhecer os valores de temperaturas abaixo e acima dos quais o desenvolvimento de cada espécie cessa;

(c) Outros fatores que afetam estimativas de tempo de desenvolvimento: muitos fatores podem afetar essa estimativa, incluindo o método de coleta das larvas no cadáver, condições microclimáticas, roupas, presença de drogas, venenos ou medicamentos no cadáver etc.

(3) Estudos sobre ecologia e padrões de sucessão na fauna cadavérica. Os dados disponíveis sobre esse assunto são extremamente precários, por serem difíceis e demorados e geralmente realizados com um animal modelo, sendo o mais comum o porco doméstico, mas comumente com poucas repetições e sem considerar a variação sazonal e a variação entre anos diferentes; um estudo realizado na Austrália (Archer 2003) mostrou que podem ocorrer grandes variações temporais no padrão de sucessão, levando a grande margem de erro nas estimativas de tempo com base em padrões de sucessão; além disso, o padrão de sucessão varia muito geograficamente, porque cada região tem uma fauna distinta. Ou seja, esses estudos devem ser realizados em todas as regiões e devem ser feitos com um bom número de repetições (modelos) em diferentes estações do ano e repetidos por vários anos para definir um padrão de sucessão.

(4) Bancos de dados. Um banco de dados sobre Entomologia Forense seria uma ferramenta extremamente útil tanto para o trabalho de pesquisa como para o trabalho de rotina dos peritos. Esse banco de dados poderia conter informações sobre os insetos de interesse forense, incluindo distribuição geográfica conhecida e informações detalhadas sobre casos e bibliografia relevante. O banco de dados deverá ser alimentado tanto por pesquisadores como por peritos.

(5) Fontes de financiamento. Os órgãos tradicionais de apoio à pesquisa, como o Conselho Nacional de Desenvolvimento Científico e Tecnológico (CNPq), as fundações de apoio à pesquisa dos Estados da Federação e o Ministério da Justiça, entre outros, têm financiado estudos de Entomologia Forense. Entretanto, seria interessante a existência de linhas específicas de demanda induzida priorizando a produção do conhecimento básico necessário para viabilizar e aperfeiçoar o trabalho de rotina dos peritos.

(6) Formação de recursos humanos. Cursos de mestrado e doutorado, universidades, têm contribuído com a formação de excelentes profissionais nas áreas de taxonomia, biologia e ecologia de insetos, entre outras. Paralelamente a esses, seriam bem-vindos cursos de curta e/ou média duração voltados ao treinamento de peritos criminais e médicos legistas acerca da importância da entomologia como meio de produção de provas materiais, no reconhecimento das diferentes apresentações dos vestígios entomológicos - estágios de desenvolvimento dos insetos - e de seus hábitos. Esses cursos serviriam a dois propósitos: (a) a tomada de consciência sobre os vestígios entomológicos pelos profissionais da polícia técnico-científica e (b) sua aproximação com os da academia. Seria altamente desejável que, para tanto, fosse estimulado, principalmente

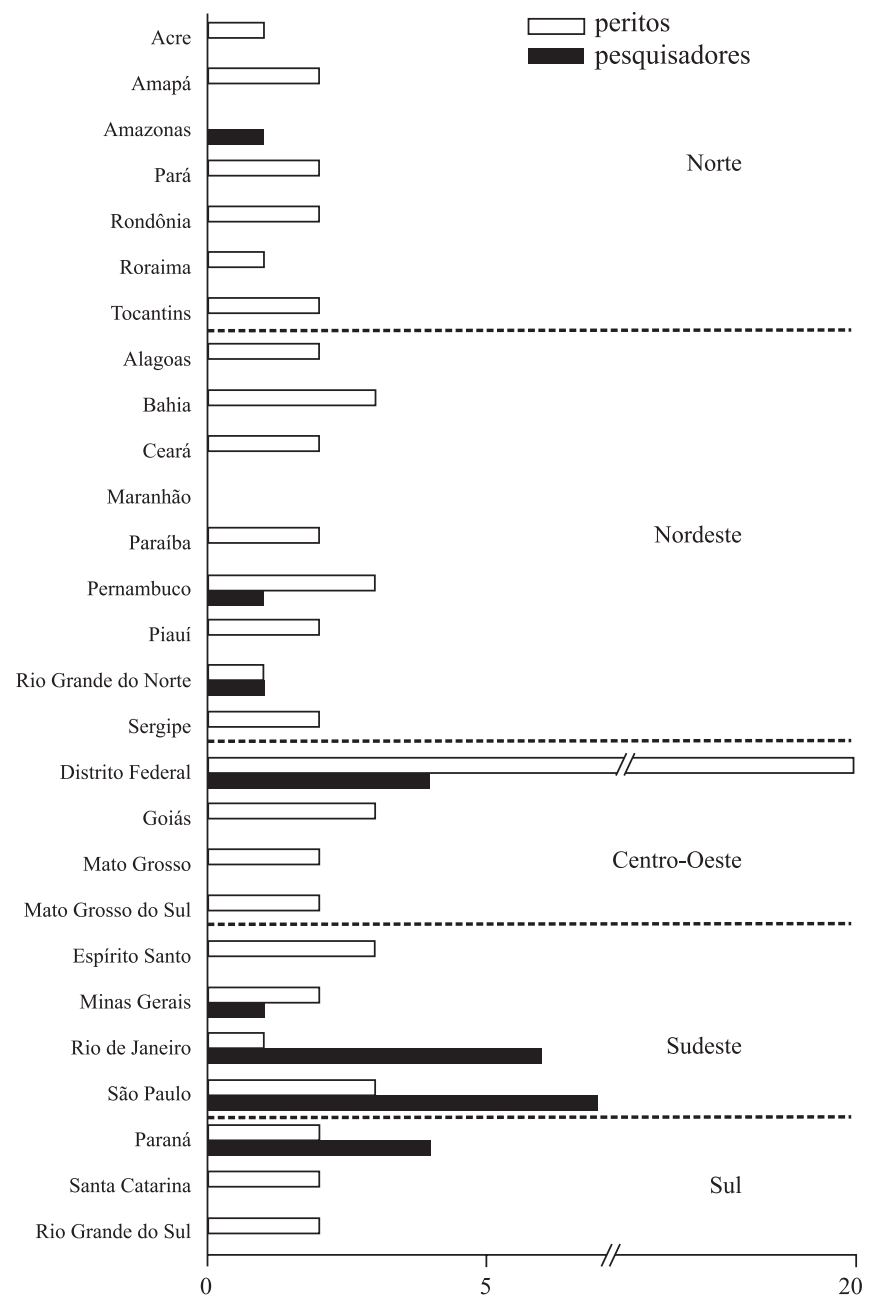

Fig. 6. Número de peritos e pesquisadores atuando em Entomologia Forense no Brasil atualmente. Fontes: ABEF (http://www.rc.unesp.br/ ib/zoologia/abef) e Plataforma Lattes (http://lattes.cnpq.br).

pelos órgãos responsáveis pelas polícias brasileiras, projetos integrados na área de educação e formação de recursos humanos. Assim como na academia, é a interação entre as atividades de magistério e pesquisa que determinam a ampliação de uma área do conhecimento. Destacamos aqui que a experiência de um entomologista em atividades de campo só pode agregar conhecimento aos estudos de criminalística se estiver intimamente associada ao conhecimento do perito criminal ou do médico legista e vice-versa. Esta confiança seria muito mais estimulada nas atividades acadêmicas de ensino e pesquisa.

(7) Publicação de uma revista especializada nas áreas de Ciências Forenses.

Agradecimentos. Os autores receberam apoio do Ministério da Justiça, da Secretaria Nacional de Segurança Pública (SENASP), do Ministério da Ciência e Tecnologia, do Conselho Nacional de Desenvolvimento Científico e Tecnológico [CNPq; processos números 308636/2007-4 (JRPL) e 307605/2007-8 (RC)]. Agradecemos a Cleide Costa, Carlos J. E. Lamas e Carlos R. F. Brandão pelo auxílio na obtenção da bibliografia e fotografias, a Nelson Papavero, Cristiane Assis-Pujol e Claudio José de Barros Carvalho pela revisão do manuscrito. 


\section{REFERÊNCIAS}

Amendt, J.; C. Campobasso; E. Gaudry; C. Reiter; H. LeBlane \& M. Hall. 2007. Best practice in forensic entomology - standards and guidelines. International Journal of Legal Medicine 121: 90-104.

Amendt, J.; R. Krettek \& R. Zehner. 2004. Forensic entomology. Naturwissenschaften 91: 51-65.

Archer, M. 2003. Annual variation in arrival and departure times of carrion insects at carcasses: implications for succession studies in forensic entomology. Australian Journal of Zoology 51: 569-576.

Benecke, M. 2001. A brief history of forensic entomology. Forensic Science International 120: $2-114$.

Benecke, M.; E. Josephi \& R. Zweihoff. 2004. Neglect of the elderly: forensic entomology cases and considerations. Forensic Science International 146: 195-199.

Benecke, M. \& R. Lessig. 2001. Child neglect and forensic entomology. Forensic Science International 120: 155-159.

Bergeret, M. 1855. Infanticide. Momification naturelle du cadavre. Annales d'Hygiène Publique et de Médicine Légale 4: 442-452.

Carter, D.; D. Yellowlees \& M. Tibbett. 2007. Cadaver decomposition in terrestrial ecosystems. Naturwissenschaften 94: 12-24.

Carvalho, C. J. B. de \& C. A. Mello-Patiu. 2008. Keys to the adults of the most common forensic species of Diptera in South America. Revista Brasileira de Entomologia 52: 390-406.

Carvalho, L. M. L.; P. J. Thyssen; A. X. Linhares \& F. A. B Palhares. 2000. A checklist of arthropods associated with pig carrion and human corpses in Southeastern Brazil. Memórias do Instituto Oswaldo Cruz 95: 135-138.

Crosby, T.; J. Watt; A. Kistemaker \& P. Nelson. 1986. Entomological identification of the origin of imported Cannabis. Forensic Science Society 26: 35-44.

Efremov, E. 1940. Taphonomy: new branch of paleontology. Pan American Geology 74: 81-93.

Freire, O. 1914a. Algumas notas para o estudo da fauna cadavérica da Bahia. Gazeta Médica da Bahia 46: 110-125.

Freire, O. 1914b. Algumas notas para o estudo da fauna cadavérica da Bahia. Gazeta Médica da Bahia 46: 149-162.

Freire, O. 1923. Fauna cadavérica brasileira. Revista de Medicina 34: $15-40$

Greenberg, B. 1991. Flies as forensic indicators. Journal of Medical Entomology 28: 565-577.

Guimarães, J.; N. Papavero \& A. Prado. 1983. As miíases na região Neotropical (identificação, biologia, bibliografia). Revista Brasileira de Zoologia 1: 239-416.

Haglung, W. \& M. Sorg. 1997. Introduction to forensic taphonomy, p. 1-26. In: W. D. Haglung \& M. H. Sorg (eds.). Forensic Taphonomy: The post mortem fate of human remains. CRC. Press, Boca Raton, xiii+684 p.

Haskell, N.; R. Hall; V. Cervenka \& M. Clark. 1997. On the body: Insects' life stage presence and their postmortem artifacts, p. 415448. In: W. D. Haglung \& M. H. Sorg (eds.). Forensic Taphonomy: The post mortem fate of human remains. CRC. Press, Boca Raton, xiii+684 p.

Keh, B.,1985. Scope and applications of forensic entomology. Annual Review of Entomology 30: 137-154.

Leclercq, M. 1969. Entomological Parasitology. The Relations between Entomology and the Medical Sciences. New York, Pergamon, $158 \mathrm{p}$.

Lord, W. D. \& J. R. Stevenson. 1986. Directory of forensic entomologists. 2 ed. Misc. Publ. Armed Forces Pest Mgt. Board, Washington, D.C, 42 p.

Lüderwaldt, G. 1911. Os insetos necrófagos paulistas. Revista do Museu Paulista 8: 414-433.

Mattos, W. B. 1919. As sarcophagas de São Paulo (These, Faculdade de Medicina e Cirurgia de São Paulo), iii + $116+$ xii pp., 3 pls. São Paulo.

Mégnin, J. 1894. La faune des cadavres: application de l'entomologie a la medecine legale. Encyclopedie Scientifique des Aides Memoires. Masson et Gauthiers-Villars, Paris, 214 p.

Miranda, G.; G. Jacques; M. P. Almeida \& M. S. B. Silva. 2006. Coleta de amostras de insetos para fins forenses. Brasília, Ministério da Justiça, iv + $11 \mathrm{p}$.

Monteiro-Filho, E. A. \& J. Penereiro. 1987. Estudo da decomposição e sucessão sobre uma carcaça animal numa área do estado de São Paulo, Brasil. Revista Brasileira de Biologia 47: 289-295.

Morton, R. J. \& W. D. Lord. 2006. Taphonomy of child-sized remains: A study of scattering and scavenging in Virginia, USA. Journal of Forensic Sciences 51: 475-479.

Moura, M. O; C.J.B. de Carvalho \& E. L. A Monteiro Filho. 1997. A Preliminary Analysis Of Insects Of Medico-Legal Importance In Curitiba, State Of Paraná. Memórias do Instituto Oswaldo Cruz 92: 269-274.

Mumcuoglu, K.; N. Gallili; A. Reshef; P. Brauner \& H. Grant. 2004. Use of human lice in forensic entomology. Journal of Medical Entomology 41: 803-806.

Oliveira-Costa, J. 2003. Entomologia Forense - Quando os insetos são vestígios. Campinas, Millennium, $257 \mathrm{p}$.

Oliveira-Costa, J. \& S. Lopes. 1999. A relevância da entomologia forense para a perícia criminal na elucidação de um caso de suicídio. Entomologia y Vectores 7: 203-209.

Oliveira-Costa, J. \& C. A. Mello-Patiu. 2004. Estimation of PMI in homicide investigation by the Rio de Janeiro Police. Aggrawal's Internet Journal of Forensic Medicine and Toxicology 5: $40-44$.

Pessôa, S. \& F. Lane. 1941. Coleópteros de interesse médico-legal. Ensaio monográfico sobre a família Scarabaeidae de S. Paulo e regiões vizinhas. Arquivos de Zoologia do Estado de São Paulo 2: $389-504$.

Pujol-Luz, J.; P. Francez; A. Ururahy-Rodrigues \& R. Constantino. 2008. The black-soldier fly, Hermetia illucens (Diptera, Stratiomyidae), used to estimate the postmortem interval in a case in Amapá State, Brazil. Journal of Forensic Sciences 53: 476-478.

Pujol-Luz, J. R.; H. Marques; A. Ururahy-Rodrigues; J. A. Rafael; F. H. Santana; L. C. Arantes \& R. Constantino. 2006. A forensic entomology case from the Amazon rain forest of Brazil. Journal of Forensic Sciences 51: 1151-1153.

Repogle, J.; W. D. Lord; B. Bodowle; T. Meinking \& D. Taplin. 1994. Identification of host DNA by amplified fragment length polymorphism (AMP-FLP) analysis of human crab louse excreta. Journal of Medical Entomology 31: 686-690.

Roquette-Pinto, E. 1908. Nota sobre a fauna cadavérica do Rio de Janeiro. A Tribuna Médica 21: 413-417.

Salviano, R.; R. Mello; R. Santos; L. Beck \& A. Ferreira. 1996. Calliphoridae (Diptera) associated with human corpses in Rio de Janeiro, Brazil. Entomologia y Vectores 3: 145-146.

Sorg, M. \& W. Haglund. 2002. Advancing forensic taphonomy: Purpose, theory, and process, p. 3-29. In: W. Haglund \& M. Sorg (eds.). Advances in forensic taphonomy. Method, theory, and archeological perspectives. CRC. Press, Boca Raton, xxv+552p.

Souza, A. M. \& A. X. Linhares 1997. Diptera and Coleoptera of potential forensic importance in Southeastern Brazil: Relative abundance and seasonality. Medical and Veterinary Entomology 11: $8-12$.

Thompson, F. \& A. C. Pont. 1993. Systematic database of Musca Names (Diptera). A catalog of names associated with the genus-group name Musca Linnaeus, with information on their classification, distribution, and documentation. Thesis Zoologicae 20. Koenigstein, Koeltz Scientific Books, 221 p.

Ubelaker, D. H. 1997. Taphonomic applications in forensic anthropology, p. 77-90. In: W. Haglund \& M. Sorg (eds.). Forensic Taphonomy: The post mortem fate of human remains. CRC. Press, Boca Raton, xxv+552 p.

Ururahy-Rodrigues, A.; H. Marques; J. Rafael; R. Wanderley \& J. PujolLuz. 2008. Coprophanaeus lancifer (Coleoptera, Scarabaeidae) activity causes the rolling movement of a man-sized carcass in Amazonia, Brazil: A forensic taphonomy report. Forensic Science International 182: 19-22.

Recebido em 09/05/2008; aceito em 29/09/2008 